\title{
Rugby seven femenino en el centro-sur de Chile: asociación entre fuerza explosiva, velocidad, agilidad y estado nutricional \\ Women's rugby seven in south-central Chile: association between explosive strength, speed, agility and nutritional status \\ *Natalia Olivera Medina, **Jaime Vásquez-Gómez \\ *Universidad San Sebastián (Chile), **Universidad Católica del Maule (Chile)
}

Resumen. Las investigaciones en el rugby seven consideran demandas durante el juego, antropometría y aptitud física. En Chile este deporte no es profesional. El objetivo fue evaluar las posibles diferencias entre el rendimiento en pruebas físicas y características antropométricas, y asociar este rendimiento con el estado nutricional en jugadoras de rugby seven del centrosur de Chile. Participaron 38 jugadoras adultas de tres equipos no profesionales, se les midió características antropométricas con balanza y estadiómetro, test de salto vertical con alfombra de saltos, velocidad y agilidad con fotocélulas en pruebas de campo. Para comparar los tres equipos se utilizó ANOVA de un factor y tamaño del efecto, la asociación se determinó con regresión lineal y coeficiente beta. Se utilizó el programa STATA v. $14(p<.05)$. Hubo diferencias entre los tres equipos en los saltos countermovement jump (CMJ), squat jump (SJ), velocidad en 30 metros y test de agilidad de Illinois $(p<.05)$ con tamaños del efecto generalmente altos, y diferencias en el índice de masa corporal (IMC) $(p=.015)$ con tamaños del efecto bajo, moderado y alto. Cuando existió mayor IMC hubo menos probabilidades de aumentar el rendimiento en CMJ, SJ, velocidad en 30 metros y agilidad, y al ajustar por variables de confusión estas probabilidades fueron menores $(p<.05)$. Ante las diferencias en los saltos, velocidad y agilidad, y a la relación inversa de estas con el IMC se sugiere mantener un nivel preparación que permita mejorar el rendimiento del rugby seven femenino en la zona.

Palabras clave: Rugby seven, deportes, femenino, aptitud física, estado nutricional.

\begin{abstract}
Research in rugby seven considers demands during the game, anthropometry and physical fitness. In Chile this sport is non-professional. The aim was to evaluate the possible differences between performance in physical tests and anthropometric characteristics, and to associate this performance with the nutritional status in rugby seven players from south-central Chile. 38 adult players from three non-professional teams participated, anthropometric characteristics was measured with scale and stadiometer, vertical jump test with jumping mat, speed and agility with photocells in field tests. One-way ANOVA and effect size were used to compare the three teams, and the association was determined with linear regression using the beta coefficient. The STATA v. 14 program was used $(p<.05)$. There were differences between the three teams in the countermovement jump (CMJ), squat jump (SJ), 30-meter sprint, and Illinois agility test $(p<.05)$ with generally high effect sizes, and differences in the body mass index (BMI) $(p=.015)$ with low, moderate, and high effect sizes. When there was a higher BMI, there were less probabilities of increasing performance in CMJ, SJ, 30-meter speed and agility, and when adjusting for confounding variables, these probabilities were lower $(p<.05)$. Given the differences in jumping, speed and agility, and the inverse relationship of these with the BMI, it is suggested to maintain a level of preparation that allows improving the performance of women's rugby seven in the area.
\end{abstract}

Key words: Rugby seven, sports, female, physical fitness, nutritional status.

\section{Introducción}

El rugby seven es un deporte de tipo intermitente y de contacto, se desarrolla en dos tiempos de siete minutos cada uno y lo pueden practicar jugadores de diversas edades y nivel competitivo (Sant'Anna, et al. 2019). En el año 2016 este deporte fue incluido en los Juegos Olímpicos de Río de Janeiro (Fuller, Taylor, \&

Fecha recepción: 07-06-20. Fecha de aceptación: 19-08-21 Jaime Andrés Vásquez-Gómez jvasquez@ucm.cl
Raftery, 2017; Misseldine, Blagrove, \& Goodwin, 2021). En el caso de Chile, el año 2004 se creó la primera selección nacional de rugby seven femenino y el año 2016 aumentaron el número de clubes para esta categoría, sin embargo, la participación de las mujeres ha sido bastante tardía respecto a los hombres (Antón, 2011).

Algunos trabajos de investigación afirman que la evaluación de las capacidades físicas y la composición corporal de cada jugadora puede ir en mejora del rendimiento físico (Báez-San Martín, et al. 2019; García, et al. 2014; Hene, 2011), ya que estos indicadores se utilizan para determinar perfiles de rendimiento 
(O’Donoghue, 2005). Es así como las capacidades físicas son importantes en el desarrollo de un partido, las que van a depender de los diferentes puestos de juego (Renedo, et al. 2006), considerando que el rugby seven se caracteriza por un alto grado de contacto físico (Clarke, Anson, \& Pyne, 2015a) siendo necesario que los jugadores sean fuertes, potentes, con resistencia para realizar aceleraciones, desaceleraciones, cambios de sentido, placajes, rucks (Rizi, et al. 2017), paradas repentinas y sprints (Lopez, et al. 2012). Estas capacidades son componentes a considerar dentro de la preparación física (Avila, 2012) ya que se relacionan con las opciones de victoria en un partido de rugby seven (Gabbett, 2005). Algunas investigaciones han informado que después de los partidos las funciones musculares disminuyen y existe un aumento de marcadores sanguíneos que evidencian daño muscular (Clarke, et al. 2015a), de manera que es importante el estudio del desempeño físico y fisiológico lo que ayudará a la elección adecuada de pruebas físicas y en la elaboración de programas de entrenamiento en el rugby seven femenino (Suarez-Arrones, et al. 2012).

La antropometría es necesaria para mejorar el rendimiento deportivo del jugador, ya que a partir de ella es posible determinar la composición corporal específica y/o global (Avila, 2012). Dentro de la antropometría las medidas más comunes son la estatura, el peso corporal y los pliegues cutáneos (Agar-Newman, Goodale, \& Klimstra, 2017). El IMC es el índice antropométrico más utilizado para la evaluación del estado nutricional (Ayvaz \& Çimen, 2011), ya que hace referencia de manera indirecta sobre la composición corporal en deportistas que tienen una mayor cantidad de masa magra, los que podrían llegar a clasificarse como sobrepeso (Carbajal, 2013). En rugbistas y en el rugby seven tener un IMC elevado, tamaño, peso corporal y buena estatura traen beneficios en las funciones de scrum, line out, y otras (Gavarry, et al. 2018; King, Hills, \& Blundell, 2005; Sella, et al. 2019), de manera que estas variables morfológicas varían según el rol posicional de las jugadoras en el campo, es decir, un menor IMC y peso corporal es característico de las backs, y valores más altos en las forwards (Sella, et al. 2019; Weinmeister, 2013). Sin embargo, se debe considerar que un mayor tejido adiposo dificultará las aceleraciones y producirá mayor gasto energético (Báez-San Martín, et al. 2019).

El énfasis debe estar puesto en la preparación física, las cualidades físicas, así como también en las medidas antropométricas, ya que mayor información se tendrá para evaluar, monitorear, guiar el proceso de entrena- miento y verificar los cambios a través de la temporada (Agar-Newman, et al. 2017; Clarke, et al. 2014), sobre todo teniendo en cuenta que la mayoría de las investigaciones en el rugby seven en la última década se han realizado en países de Europa, en Australia, Nueva Zelandia, Sudáfrica, Kenia, Norteamérica, China, Japón, Reino Unido, y muy poco en América Latina como es el caso de Colombia y Brasil (Lopez, et al. 2012; Sella, et al. 2019) y como consecuencia existe poco conocimiento sobre las demandas del juego en el rugby seven femenino (Misseldine, et al. 2021). La versión femenina de este deporte tiene la cualidad de ser de tipo aficionado, a nivel universitario ha reportado ser emergente (Rizi, et al. 2017) y en Chile tiene un tenor no profesional de tipo amateur más que competitivo, por lo que la aptitud física de las jugadoras puede variar durante una temporada debido a la cantidad y nivel de las sesiones de entrenamiento, del nivel de competencia y variables sociales, entre otras (Hene, 2011). Por tanto, el objetivo de este trabajo fue evaluar posibles diferencias en el rendimiento en pruebas físicas y las características antropométricas entre tres equipos de rugby seven femenino del centro-sur de Chile, y evaluar la asociación entre las pruebas físicas y el estado nutricional.

\section{Material y método}

Se utilizó un diseño no experimental, de alcance descriptivo y correlacional. El muestreo fue no probabilístico en que participaron mujeres deportistas mayores de 18 años de tres equipos de rugby seven de tipo aficionado del centro-sur de Chile. Los tres equipos, R7F1, R7F2 y R7F3 tuvieron un total de 12, 12 y 14 participantes, respectivamente. Para acceder al estudio las deportistas no debieron tener lesiones musculoesqueléticas para realizar los test físicos y firmar por escrito un consentimiento ad hoc de participación. No fue necesaria la aprobación de un comité de ética porque los datos se obtuvieron como parte de las evaluaciones rutinarias durante el ciclo de entrenamiento anual (Winter \& Maughan, 2009). La caracterización y comparación entre los tres equipos se aprecia en la tabla 1 .

Se midió el peso corporal y la estatura con una balanza digital OMRON modelo HBF-214m-LA con capacidad máxima $150 \mathrm{~kg}$ (Japón) y un estadiómetro

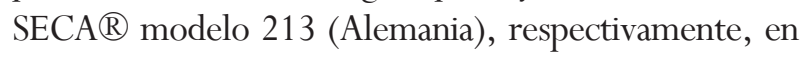
que las deportistas tuvieron que estar descalzas y con ropa ligera (pantalón corto, calza y polera, peto deportivo), y con estas mediciones se calculó el IMC. La fuer- 
za explosiva de tren inferior se midió a través de los saltos verticales CMJ (González, et al. 2014) y SJ (dal Pupo, Detanico, \& dos Santos, 2012) en los que se registró la altura en centímetros y la potencia en vatios (interacción entre el peso corporal, la altura de salto y tiempo de vuelo), para ello se utilizó una alfombra de saltos DM-JUMP $\AA$ modelo by Prometheus ${ }^{\circledR}$ (Chile) y un ordenador Toshiba, modelo Satéllite $\mathbb{R}$ L45-A (Chile) con el software V2.3.5 Beta compatible con la alfombra de saltos. La velocidad horizontal se midió con el test de carrera en 30 metros desde salida alta y con el extremo anterior del pie de elección justo detrás de la línea de inicio (Oyarzo, Said, \& Nazar, 2017), para esta prueba se midió la distancia a recorrer con una cinta métrica de 30 metros (Redline $\AA$, EE. UU.) delimitando el inicio y fin del trayecto con conos en los extremos. El tiempo se midió de manera digital utilizando fotocélulas (Microgate $\AA$, modelo Polifemo Radio SF, Italia) registrándose los datos en segundos, con dos decimales. Para medir la agilidad se utilizó el test de Illinois (Raya, et al. 2013) en donde el tiempo también se midió de forma digital con fotocélulas registrándose en segundos y con dos decimales. El test de Illinois se inició desde tendido en pronación y se pasó de forma rápida a la posición de pie, se siguió con acciones multidireccionales las que estuvieron delimitadas por obstáculos.

En la primera sesión se midió las características antropométricas y los test de salto vertical en una sala en condiciones adecuadas, en la segunda sesión se aplicó el test de velocidad y el de agilidad en terreno de juego. Cada test de rendimiento físico se realizó 3 veces, la pausa fue de 1 a 3 minutos entre cada intento, con una pausa completa de 5 a 8 minutos entre cada test, y se computó el valor promedio.

Las variables continuas se presentaron en valores promedio y desviación estándar, y se sometieron a la prueba de distribución normal de Shapiro-Wilk. Para comparar las mediciones entre los tres grupos se utilizó la prueba ANOVA de un factor con la prueba post hoc de Tukey, y se calculó el tamaño del efecto con la $d$ de Cohen entre los tres grupos considerando los siguientes valores: $<.2$ trivial; $.2-.5$ bajo; $.5-.8$ moderado; $>.8$ alto. La asociación entre la fuerza explosiva, velocidad, agilidad y el IMC se determinó con regresión lineal calculando el coeficiente beta $(\beta)$ con intervalos de confianza (IC) del 95\%, y se elaboraron tres modelos ajustados por variables de confusión. El análisis de datos se realizó con el programa estadístico STATA v.14 y se asumió una significación estadística con un $p$-valor $<.05$.

\section{Resultados}

En la tabla 1 se aprecia que para la estatura solo difirieron significativamente el equipo uno con el tres, y en el IMC el equipo dos con el tres. Así, el entrenamiento que han llevado los tres equipos antes de hacer las mediciones, más aspectos nutricionales, laborales, etc. que pudieron influir en los resultados y que no se consideraron en este estudio, demostró que la comparación del IMC entre los tres equipos tuvo un tamaño del efecto bajo, moderado y alto. Por su parte, ya que no hubo diferencias en la edad y en el peso corporal, la magnitud del efecto fue trivial y bajo, y desde trivial a moderado, respectivamente.

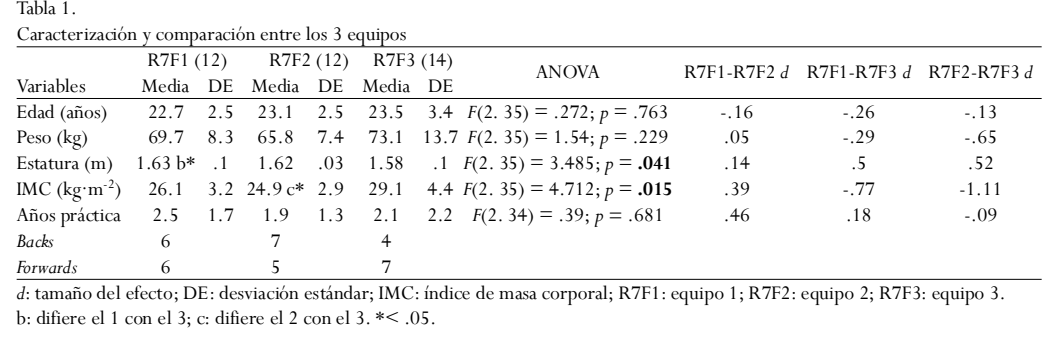

Caracterización y comparación entre los 3 equipos

F3 $d$ R7F2-R7F3d : tamaño del efecto; DE: desviación estándar; IMC: índice de masa corporal; R7F1: equipo 1; R7F2: equipo 2; R7F3: equipo 3. b: difiere el 1 con el 3 ; c: difiere el 2 con el $3 . *<.05$.

Respecto al rendimiento en los saltos verticales (CMJ y SJ) se encontraron diferencias entre los tres equipos, salvo para el SJ medido en vatios. Es constante que dichas diferencias fueron entre el equipo uno y tres, $y$ entre el dos y el tres, siendo los equipos uno y dos con los valores promedios más altos registrados, y con tamaños del efecto altos en gran parte de las comparaciones entre equipos. En los otros test también existieron diferencias significativas, en la velocidad en 30 metros solo difirió el equipo uno con el tres, en el test de Illinois fueron distintos el uno con el tres y el dos con el tres, y en las comparaciones la mayoría de ellos arrojaron tamaños del efecto altos (Tabla 2).
Tabla 2.

Comparación entre los test de salto, velocidad y agilidad

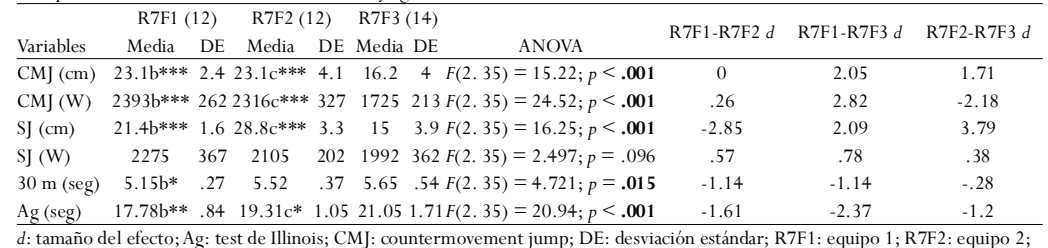
R7F3: equipo 3; SJ: squat jump; seg: segundos; W: vatios; $30 \mathrm{~m}$ : velocidad en 30 metros.

a: difiere el 1 con el 2 ; b: difiere el 1 con el 3 ; c: difiere el 2 con el $3 . *<.05 ; * *<.01 ; * *<.001$ 
En la tabla 3 se puede apreciar una asociación inversa entre los saltos verticales y el IMC, es decir, cuando las deportistas tuvieron un mayor IMC existieron significativamente menos probabilidades de aumentar el rendimiento en CMJ y SJ, aunque esta interacción no fue significativa para el SJ medido en vatios. Cuando el modelo 1 se ajustó por edad las probabilidades de incrementar el rendimiento en los saltos verticales fueron menores, y al ajustar por años de práctica y posición de juego estas probabilidades siguieron decreciendo. Comportamiento similar tuvieron los modelos que incluyeron la velocidad de desplazamiento como variable de exposición, a mayor IMC se presentó un peor rendimiento en la velocidad en 30 metros y en la agilidad (hacer un mayor tiempo de ejecución). Al ajustar el modelo 1 por la edad no hubo variaciones, pero sí las hubo al ajustar por años de práctica y posición de juego (las jugadoras tuvieron $2.2 \pm 1.8$ años de práctica, y como posición de juego 17 fueron backs y 18 forwards).

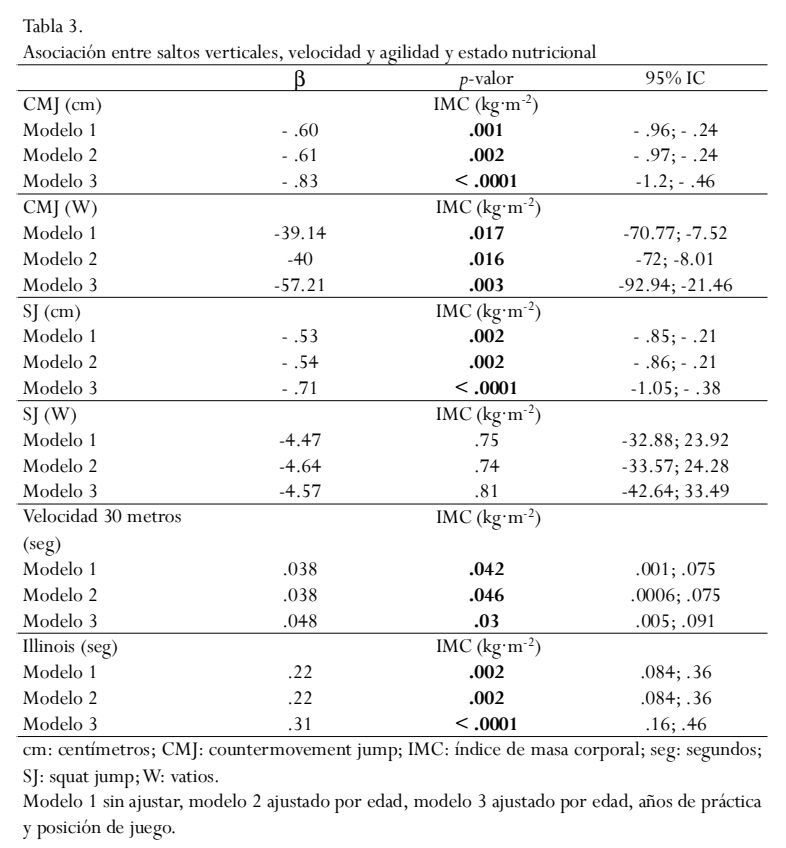

\section{Discusión}

Como principal resultado de esta investigación podemos señalar que los saltos verticales, velocidad de carrera y agilidad estuvieron influenciados por el estado nutricional, es decir, a mayor IMC hubo menos probabilidades de mejorar el rendimiento en las pruebas físicas, y cuando se añadió variables demográficas como la edad y variables sobre la práctica del rugby seven estas probabilidades fueron aún menores.

Las investigaciones en el rugby seven femenino se han centrado en variables sobre las demandas del juego (Sella, et al. 2019), cualidades físicas y características antropométricas (Agar-Newman, et al. 2017; Sella, et al. 2019), y mayormente en estas dos últimas en jugadoras no profesionales o de no-élite (Sella, et al. 2019). Respecto a las cualidades físicas, las jugadoras de nuestro estudio que pertenecieron al equipo R7F1 y R7F2 tuvieron $23.1 \mathrm{~cm}$ en el test CMJ, y el equipo R7F2 obtuvo $28.8 \mathrm{~cm}$ en la prueba SJ. Estos valores, que fueron los registros más altos, han sido menores a los reportados en jugadores de rugby XV (CMJ: 33.6 y SJ: $30.2 \mathrm{~cm}$ ) (Nápoli, 2016) y en jugadores de rugby seven (CMJ: 28.73 a $36.02 \mathrm{~cm}$ ) (Martins, et al. 2018). Estas diferencias entre ambos sexos ya han sido explicadas en la literatura debido a las características biológicas y hormonales (García \& Landa, 2005). También se han encontrado registros mucho más altos (cercanos y mayores a $50 \mathrm{~cm}$ ) en jugadoras de rugby seven no profesionales y jugadores universitarios, pero en estos estudios no se ha detallado el tipo de test de salto vertical aplicado (Clarke, Anson, \& Pyne, 2017; Rizi, et al. 2017).

El tiempo alcanzado en la distancia de 30 metros por las jugadoras de nuestra investigación (desde 5.15 hasta 5.65 segundos) ha sido mayor comparado con jugadoras amateur (Mina, 2018), pero también mejores que los registros de jugadoras del tipo no-élite, profesionales y jugadores universitarios de rugby seven que recorrieron distancia de 40 metros inclusive (AgarNewman, et al. 2017; Clarke, et al. 2017; Rizi, et al. 2017). Respecto al test de agilidad los resultados son dispares, ya que por un lado se ha encontrado mejor desempeño en nuestras deportistas (menor tiempo: de 17.7 a 21 segundos) respecto a jugadoras de rugby seven de élite (Nyberg \& Penpraze, 2016), menor rendimiento (mayor tiempo) comparado con jugadores de categoría senior (Gabbett, 2002) y con jugadores de nivel universitario (Rizi, et al. 2017). Estos contrastes pueden deberse al nivel de entrenamiento y nivel competitivo de los deportistas, diferencias que se han confirmado al comparar jugadoras de nivel profesional y no-élite respecto al rendimiento en test de velocidad y saltos verticales (Sella, et al. 2019).

La evidencia comparada nos indicó que fue pertinente realizar pruebas de velocidad, que en la literatura crítica sobre la disciplina abarcan desde los 10 hasta los 40 metros, y con test de saltos, preferentemente del tipo vertical, para la evaluación de línea de base de las jugadoras de rugby seven. También la evidencia comparada reportó que este tipo de prestaciones físicas formó parte importante de las baterías de pruebas para estas deportistas. Por tanto, lo estudiado con las deportistas chilenas se adecuó al estado del arte en el diagnós- 
tico y monitoreo de esta disciplina. En este sentido, es importante destacar que los resultados en los test de salto vertical incidieron en las pruebas de velocidad y agilidad desarrollados por las jugadoras chilenas, es decir, cuando se logró mayor altura en los test de salto hubo menor tiempo de registro en velocidad de 30 metros y test de Illinois, y a la vez existió una asociación entre el test de 30 metros y el de agilidad. Esta relación entre fuerza explosiva y velocidad también ha sido demostrada en la literatura clásica (Cometti, 2002; Galvis, Arabia, \& Castro, 2007).

Respecto a las características antropométricas estudiadas en el rugby seven, el peso corporal de las jugadoras se ha mantenido estable (de 66.4 a $67.8 \mathrm{~kg}$ ) en las últimas ediciones de los torneos de clasificación 2014/ 15 y 2015 / 16 y en los Juegos Olímpicos de Río de Janeiro 2016 (Fuller, Taylor, \& Raftery, 2017), aunque con algunas variaciones en los últimos años (66.4 a 72.8) (AgarNewman, et al. 2017). En jugadoras no profesionales ha tenido un rango entre $53.3 \pm 5.1$ y $71.7 \pm 13.9 \mathrm{~kg}$ (Clarke, Anson, \& Pyne, 2015b; Clarke, et al. 2015a, 2017; Leite, et al. 2016; Ma, et al. 2016; Rizi, et al. 2017; Portillo, et al. 2014) y se han encontrado hallazgos similares $(66.8 \mathrm{~kg})$ en jugadoras no profesionales, que al ser comparado con jugadoras de élite han tenido un tamaño del efecto pequeño (Sella, et al. 2019). Las jugadoras chilenas de este estudio reportaron pesos corporales entre 65.8 y $73.1 \mathrm{~kg}$ lo que va en un rango similar a lo informado en la literatura recién expuesta. El peso corporal de las jugadoras podría hipotéticamente interferir en el rendimiento de los test físicos, al considerarlo de forma aislada y sin interacción con la estatura.

Cuando se evaluó el estado nutricional de las jugadoras del equipo R7F1 estas tuvieron $26.1 \mathrm{~kg} \cdot \mathrm{m}^{-2}$, el $\mathrm{R} 7 \mathrm{~F} 2$ un valor de $24.9 \mathrm{~kg} \cdot \mathrm{m}^{-2}$ y R7F3 de $29.1 \mathrm{~kg} \cdot \mathrm{m}^{-2}$ en el IMC, que indicó sobrepeso para el primer y tercer equipo. También se ha estudiado a jugadoras de rugby seven aficionadas y se ha encontrado sobrepeso y normopeso $\left(27.5 \mathrm{~kg} \cdot \mathrm{m}^{-2}\right.$ y $23.3 \mathrm{~kg} \cdot \mathrm{m}^{-2}$ ) (de Sousa, et al. 2016), normalidad en el IMC en jugadoras jóvenes chilenas (Lagos-Hernández, et al. 2018) y sobrepeso en rugbistas de la zona central de Chile (Báez-San Martín, et al. 2019). Estos resultados son similares a los encontrados en las jugadoras estudiadas en la presente investigación, aunque se debe considerar que generalmente el IMC se presenta con valores elevados en este tipo de deportistas lo que de alguna manera es deseable para la disciplina, por tanto, es apresurado catalogarlo como desfavorable. Ante esto, se ha recomendado comple- mentar el estudio del IMC con mediciones de la masa grasa y masa libre de grasa (Gavarry, et al. 2018). Por su parte, si bien el IMC se clasificó como sobrepeso en algunas jugadoras chilenas (R7F1 y R7F3) es conveniente mantenerlo en un estado que permita un desempeño adecuado de la fuerza explosiva, la velocidad y la agilidad para la categoría no profesional, así como lo han mostrado los modelos de regresión presentados en los resultados de nuestra investigación.

Una fortaleza de este estudio fue la utilización de instrumentos fiables y válidos como la alfombra de salto, células fotoeléctricas y test de campo, lo que brindó la posibilidad de caracterizar un perfil de rendimiento físico de estas jugadoras de rugby seven del centro-sur de Chile que podrá servir de referencia para nuevas investigaciones. Por su parte, una limitación fue el bajo número de la muestra, lo que sugiere tomar con cautela la interpretación de los resultados.

\section{Conclusiones}

Se concluye que existieron diferencias significativas en la estatura e IMC entre los tres equipos de jugadoras de rugby seven, con tamaños del efecto entre trivial y alto. Lo mismo ocurrió con la mayoría de los test de salto vertical, test de velocidad y agilidad, con tamaños del efecto generalmente altos. Por último, el IMC tuvo una asociación negativa o inversa con el rendimiento de la mayoría de los test de salto vertical, con el desempeño en el test de velocidad y agilidad, demostrando que a mayor IMC existió una merma en el rendimiento. Por tanto, se sugiere mantener un nivel de IMC que permita mejorar el rendimiento en estas pruebas físicas para que estas variables ayuden a lograr un mayor rendimiento físico y nivel de competición en la zona, aunque es recomendable utilizar con criterio el IMC como herramienta de evaluación nutricional debido a sus limitaciones en la discriminación de tejidos (masa grasa y masa libre de grasa).

\section{Agradecimientos}

Agradecemos a todas las deportistas participantes del estudio, a los entrenadores y encargados de llevar a cabo el rugby seven en la zona.

\section{Referencias}

Agar-Newman, D. J., Goodale,T. L., \& Klimstra, D. (2017). Anthropometricand physical qualities of international level 
female rugby sevens athletes based on playing position. Journal of Strength and Conditioning Research, 31(5), 13461352. https://doi.org/10.1519/ JSC.0000000000001167

Antón, E. (2011). El rugby: historia y aplicación en la educación física. Pedagogía Magna, 1(11), 90-97.

Avila, O. B. (16-19 de octubre de 2012). Análisis discriminante: aplicación a la clasificación de jugadores de rugby según puestos de juego [Disertación]. Décimo Congreso Latinoamericano de Sociedades de Estadística. Ciudad Universitaria, Universidad Nacional de Córdoba, Argentina.

Ayvaz, G., \& Çimen, A. R. (2011). Methods for body composition analysis in adults. Open Obesity Journal, 3(1), 62-69.

Báez-San Martín, E., Jil-Beltrán, K., Ramírez-Campillo,Tuesta, M., Barraza-Gómez, F., Opitz-Ben-Hour, A.,... \& Yáñez-Sepúlveda, R. (2019). Body composition and somatotype of chilean rugby players and their relationship with the game position. International Journal of Morphology, 37(1), 331-137.

Carbajal, A. (12 de septiebre de 2013). Manual de Nutrición y Dietética. Recuperado de https://eprints.ucm.es/id/ eprint/22755/

Clarke, A. C., Anson, J. M., \& Pyne, D. B. (2015a). Neuromuscular fatigue and muscle damage after a women's rugby sevens tournament. International Journal of Sports Physiology and Performance, 10(6), 808-814.https: / /doi.org/101123/ijspp.2014-0590

Clarke,A. C.,Anson, J. M., \& Pyne, D. B. (2015b).The effect of running demands and impacts on post-tournament markers of inflammation and haemolysis in women's rugby sevens. New Zealand Journal of Sports Medicine, 42(2), $70-76$.

Clarke, A. C., Anson, J. M., \& Pyne, D. B. (2017). Game movement demands and physical profiles of junior, senior and elite male and female rugby sevens players. Journal of Sports Sciences, 35(8), 727-733. https://doi.org/ 10.1080/02640414.2016.1186281

Clarke,A. C., Presland, J., Rattray, B., \& Pyne, D. B. (2014). Critical velocity as a measure of aerobic fitness in women's rugby sevens. Journal of Science and Medicine in Sport, 17(1), 144-148. https://doi.org/10.1016/ j.jsams.2013.03.008

Cometti, G. (2002). La preparación física en el fútbol. Barcelona: Paidotribo.

dal Pupo, J., Detanico, D., \& dos Santos, S. G. (2012). Parâmetros cinéticos determinantes do desempenho nos saltos verticais. Revista Brasileira de Cineantropometria e Desempenho Humano, 14(1), 41-51. https://doi.org/ 10.5007/1980-0037.2012v14n1p41

de Sousa, N. M. F., Stinguel, H., de Souza Mairink, R., Baia, D. P., Bertucci, D. R., \& Martins, R. A. S. (2016). Perfil antropométrico e aeróbio de jogadoras de uma equipe de rugby sevens: diferenças entre posições táticas. Revista Brasileira de Prescrição e Fisiologia Do Exercício (RBPFEX), 10(58), 282-289.

Fuller, C. W., Taylor, A., \& Raftery, M. (2017). 2016 Rio Olympics: an epidemiological study of the men's and women's rugby-7s tournaments. British Journal of Sports Medicine, 51(17), 1272-1278. https://doi.org/ 10.1136/bjsports-2016-097301

Gabbett,T. J. (2002). Physiological characteristics of junior and senior rugby league players. British Journal of Sports Medicine, 36(5), 334 -339. https://doi.org/10.1136/ bjsm.36.5.334

Gabbett,T. J. (2005). Science of rugby league football: a review. Journal of Sports Sciences, 23(9), 961-976. https: / / doi.org/ 10.1080/02640410400023381

Galvis, E. A. M., Arabia, J. J. M., \& Castro, C.A. C. (2007). El trabajo de fuerza en el desarrollo de la potencia en futbolistas de las divisiones menores de un equipo profesional de fútbol. Iatreia, 20(2), 127-143.

García, M., \& Landa, M. (2005). Atletismo 4:la preparación del corredor de resistencia. Libro. Madrid: Real Federación Española de Atletismo.

García, M., Martínez-Moreno, J. M., Reyes-Ortiz,A., Suarez Moreno-Arrones, L., García, A., \& Garcíacaballero, M. (2014). Cambios en la composición corporal de jugadores de rugby de alta competición durante las fases de una temporada habitual: influencia de la dieta y la carga de ejercicio. Nutricion Hospitalaria, 29(4), 913-921. https: / /doi.org/10.3305/nh.2014.29.4.7227

Gavarry, O., Lentin, G., Pezery, P., Delextrat,A., Chaumet, G., Boussuges, A., .. . \& Piscione, J. (2018). A cross-sectional study assessing the contributions of body fat mass and fatfree mass to body mass index scores in male youth rugby players. Sports Medicine - Open, 4(1), 1-9.https: / / doi.org/ 10.1186/s40798-018-0130-7

González,Y., Sedano, S., Fernández, F., \& Díaz, H. (2014). Estudio comparativo de factores antropométricos y de condición física en jugadores jóvenes de voleibol colombiano. Revista U.D.C.A Actualidad \& Divulgación Científica, 17(1), 53-63.

Hene, N. M. (2011). Physical fitness of women's rugby union players over a competition season [Tesis de Maestría, University of theWestern Cape]. Recuperado de https: / / core.ac.uk/ download/pdf/58913764.pdf

King, N. A., Hills, A. P., \& Blundell, J. E. (2005). High body mass index is not a barrier to physical activity: analysis of international rugby players' anthropometric data. European Journal of Sport Science, 5(2), 73-75. https://doi.org/ $10.1080 / 17461390500148466$

Leite, M.A. F. D. J., Sasaki, J. E., Lourenço, C. L. M., Zanetti, H. R., Cruz, L. G., Mota, G. R. D., ... \& Mendes, E. L. 
(2016). Medicine ball throw test predicts arm power in rugby sevens players. Revista Brasileira de Cineantropometria e Desempenho Humano, 18(2), 166-176. https: / /doi.org/ 10.5007/1980-0037.2016v18n2p166

Lagos-Hernández, R. I., Bruneau-Chávez, J. G., AdriazolaOjeda, C. P., Bustos-San Martín, M. B. , Leiva-Peña, C.A., \& Macías-Urra,I.A. (2018). Perfil antropométrico e imagen corporal de escolares rugbistas de sexo femenino de la Región de la Araucanía, Chile. Pensar en Movimiento: Revista de Ciencias del Ejercicio y La Salud, 16(2), e31889.

Lopez, Jr.V., Galano, G. J., Black, C. M., Gupta, A.T., James, D. E., Kelleher, K. M., . . . \& Allen,A.A.(2012). Profile of an american amateur rugby union sevens series. American Journal of Sports Medicine, 40(1), 179-184. https: / / doi.org/ 10.1177/0363546511427124

Ma, R., Lopez Jr,V.,Weinstein, M. G., Chen, J. L., Black, C. M., Gupta, A.T., ... \& Allen, A.A. (2016). Injury profile of american women's rugby-7s. Medicine and Science in Sports and Exercise, 48(10), 1957-1966. https://doi.org/ 10.1249/MSS.0000000000000997

Mina, O. (2018). Desarrollo de las capacidades de la velocidad, fuerza, resistencia y su influencia en el deporte del rugby femenino siete de la categoría sénior de 18 a 20 años en el «Club Panteras RC» de la ciudad de Quito en el año 2017 [Tesis de Licenciatura, UniversidadTécnica del Norte, Ecuador]. Recuperado de http: / / repositorio.utn.edu.ec/handle/ 123456789/8059

Martins, M. S., Nunes, E. M., Rodrigues, C. D., HernándezMosqueira, C., \& da Silva, S . F. (2018). Características antropométricas y potencia de miembros inferiores en jugadores universitarios de rugby-7. MHSALUD: Revista En Ciencias Del Movimiento Humano y Salud, 15(2), e1 . http: / /dx.doi.org/10.15359/mhs.15-2.4

Misseldine, N. D., Blagrove, R. C., \& Goodwin, J. E. (2021). Speed demands of women's rugby sevens match play. Journal of Strength and Conditioning Research, 35(1), 183 189. JSC.0000000000002638

Nápoli, M. O. (2016). Evaluación diagnóstica del perfil de rendimiento de jugadores del plantel superior de lanús rugby club [Tesis de Licenciatura]. Universidad Nacional de La Plata, Argentina. Recuperado de http:// w w w. m e moria.fah ce.unl p. ed u . ar / library? $\mathrm{a}=\mathrm{d} \& \mathrm{c}=$ tesis\&d=Jte1328

Nyberg, C. C., \& Penpraze, V. (2016). Determination of anthropometric and physiological performance measures in elite scottish female rugby union players. International Juornal of Reserch in Exercie Physiology, 12(1), 10-16.

O’Donoghue, P. (2005). Normative profiles of sports performance. International Journal of Performance Analysis in Sport, 5(1), 104-119.https://doi.org/10.1080/ 24748668.2005.11868319

Oyarzo, C.A., Said, M. J., \& Nazar, M. J. (2017). Correlación del single hop test con la prueba de velocidad en treinta metros en infantes entre diez y doce años de un colegio privado de Santiago de Chile. RETOS NuevasTendencias En Educación Física, Deporte y Recreación, 1(32), 101-105.

Portillo, J., González-Ravé, J. M., Juárez, D., García, J. M., Suárez-Arrones, L., \& Newton, R. U. (2014). Comparison of running characteristics and heart rate response of international and national female rugby sevens players during competitive matches. Journal of Strength and Conditioning Research, 28(8), 2281-2289. https:// doi.org/10.1519/JSC.0000000000000393

Raya, M. A., Gailey, R. S., Gaunaurd, I. A., Jayne, D. N., Campbell, S. M., Gagne, E., ... Tucker, C. (2013). Comparison of three agility tests with male servicemembers: edgren side step test, t-test, and illinois agility test. Journal of Rehabilitation Research and Development, 50(7), 951-960. https://doi.org/10.1682/ JRRD.2012.05.0096

Renedo, M. A., Núñez Álvarez, V. M., Da Silva, M. E., Poblador, M. S., \& Lancho, J. L. (2006). Índices antropométricos de proporcionalidad corporal de jugadores cadetes y juveniles de rugby. Archivos de Medicina del Deporte, 23(113), 195-204.

Rizi, R. M.,Yeung, S. S., Stewart, N. J., \&Yeung, E.W. (2017). Risk factors that predict severe injuries in university rugby sevens players. Journal of Science and Medicine in Sport, 20(7), 648-652. https: / / doi.org/10.1016/ j.jsams.2016.11.022

Sant'Anna, R. T., Roberts, S. P., Moore, L. J., \& Stokes, K.A. (2019). Physical demands of refereeing rugby sevens matches at different competitive levels. Journal of Strength and Conditioning Research, 33(7), e1. https://doi.org/ 10.1519/JSC.0000000000003246

Sella, F. S., McMaster, D. T., Beaven, C. M., Gill, N. D., \& Hébert-Losier, K. (2019). Match demands, anthropometric characteristics, and physical qualities of female rugby sevens athletes: a systematic review. Journal of Strength and Conditioning Research, 33(12), 3463-3474. https:/ / doi.org/10.1519/JSC.0000000000003339

Suarez-Arrones, L., Nuñez, F. J., Portillo, J., \& MendezVillanueva, A. (2012). Match running performance and exercise intensity in elite female rugby sevens. Journal of Strength and Conditioning Research, 26(7), 1858-1862. https: / / doi.org/10.1519/JSC.0b013e318238ea3e

Weinmeister, L. (25 de junio de 2013). Características antropométricas del seleccionado argentino de rugby femenino seven. Recuperado de https: / / g-se.com/caracteristicasantropometricas-del-seleccionado-argentino-de-rugbyfemenino-seven-bp-457cfb26d45537

Winter, E. M., \& Maughan, R. J. (2009). Requirements for ethics approvals. Journal of Sports Sciences, 27(10), $985-$ 985.https: / /doi.org/10.1080/02640410903178344 